\title{
DIRECT CALCULATION OF MAXIMUM LIKELIHOOD ESTIMATOR FOR THE BIVARIATE POISSON DISTRIBUTION
}

\author{
By KazUTOMo Kawamura
}

\section{Summary}

To estimate the parameter vector $\lambda$ of bivariate Poisson distribution [1], [2] we would like to calculate maximum likelihood estimator (MLE) $\hat{\lambda}$. This MLE $\hat{\lambda}$ has not a simple expression as $\bar{X}, S^{2}, \cdots$ etc. We only have information about MLE $\hat{\lambda}$ by normal equations and its variation forms [3]. Holgate [4] shows the asymptotic property of MLE $\hat{\lambda}$.

In this paper we would like to show the calculating method of MLE $\hat{\lambda}$. The method will be constructed by direct calculation of likelihood function and by a searching routine of MLE $\hat{\lambda}$ which maximizes the function value. A sequence of random numbers come from a bivariate Poisson distribution $P(\lambda)$ will be shown. The change of the value of likelihood function varying parameter $\lambda$ in our rule will be calculated and the work of the searching routine will be discussed in detail. In the last part of this paper a numerical interpretation of our routine will be shown.

\section{Section 1. Bivariate Poisson distribution $P(\lambda)$.}

If $(X, Y)$ has a bivariate distribution,

$$
P(X=k, Y=l)=\sum_{\substack{\beta+\delta=k \\ \gamma+\delta=l}} \frac{\lambda_{10}^{\beta} \lambda_{01}^{\gamma} \lambda_{11}^{\delta}}{\beta ! \gamma ! \delta !} e^{-\lambda_{10}-\lambda_{01}-\lambda_{11}}
$$

we shall call $(X, Y)$ has a bivariate Poisson distribution $P(\lambda)$, where $k, l, \beta, \gamma$ and $\delta$ are nonnegative integers and nonnegative $\lambda_{10}, \lambda_{01}$ and $\lambda_{11}$ are called as parameters and $\lambda$ means a vector of the three parameters $\left(\lambda_{10}, \lambda_{01}, \lambda_{11}\right)$.

The moment generating function of the distribution is given by

$$
g\left(s_{1}, s_{2}\right)=e^{-\left(\lambda_{10}+\lambda_{01}+\lambda_{11}\right)+\lambda_{10} s_{1}+\lambda_{01} s_{2}+\lambda_{11} s_{1} s_{2}} .
$$

And the marginal distribution is given by

Received July 7, 1983 


$$
\begin{array}{ll}
P(X=k)=p\left(k ; \lambda_{10}+\lambda_{11}\right) & (k=0,1,2, \cdots), \\
P(Y=l)=p\left(l ; \lambda_{01}+\lambda_{11}\right) & (l=0,1,2, \cdots),
\end{array}
$$

where $p(* ; \lambda)$ is an univariate Poisson density. We will get more information about the distribution from the equalities:

$$
\begin{gathered}
E(X)=\lambda_{10}+\lambda_{11}, \quad \operatorname{Var}(X)=\lambda_{10}+\lambda_{11}, \\
E(Y)=\lambda_{01}+\lambda_{11}, \quad \operatorname{Var}(Y)=\lambda_{01}+\lambda_{11}, \\
\operatorname{Cov}(X, Y)=\lambda_{11} .
\end{gathered}
$$

If $(X, Y)$ has a bivariate Poisson distribution $P(\lambda)$ then we will get the decomposition rule

$$
X=X_{10}+X_{11}, \quad Y=X_{01}+X_{11}
$$

where $X_{10}, X_{01}$ and $X_{11}$ are mutually independent univariate Poisson distributions with parameter $\lambda_{10}, \lambda_{01}$ and $\lambda_{11}$ respectively.

But getting a sample $(x, y)$ of the distribution, we do not know the decomposed samples $x_{10}, x_{01}$ and $x_{11}$ satisfying the decomposition rule of the last. two equalities. This is the main reason why it is difficult to estimate the parameters from the samples of the distribution.

\section{Section 2. MLE of the parameter $\lambda$.}

Practical estimation of the parameter $\lambda=\left(\lambda_{10}, \lambda_{01}, \lambda_{11}\right)$.

Denote $n$ independent sample variables of a bivariate Poisson distribution $P(\lambda)$ as

$$
\left(X_{1}, Y_{1}\right),\left(X_{2}, Y_{2}\right), \cdots,\left(X_{n}, Y_{n}\right)
$$

and denote the practical samples as

$$
\left(x_{1}, y_{1}\right),\left(x_{2}, y_{2}\right), \cdots,\left(x_{n}, y_{n}\right) \text {. }
$$

The maximum likelihood estimator MLE of $\lambda_{10}+\lambda_{11}$ will be given by $\sum_{\imath=1}^{n} x_{\imath} / n$ and MLE of $\lambda_{01}+\lambda_{11}$ by $\sum_{\imath=1}^{n} y_{2} / n$. To estimate the parameters $\lambda_{10}, \lambda_{01}$ and $\lambda_{11}$ individually we need to discuss the method of the estimation in a more delicate way.

2-1. Practical estimation of covariance value $\lambda_{11}$.

We shall consider the problem how to estimate $\lambda_{11}$.

THEOREM 1. From $n$ independent samples $\left(x_{1}, y_{1}\right),\left(x_{2}, y_{2}\right), \cdots,\left(x_{n}, y_{n}\right)$, MLE of $\lambda_{10}, \lambda_{01}$ and $\lambda_{11}$ is given by next three equalities,

$$
\frac{1}{n} \sum_{i=1}^{n} \frac{p\left(x_{i}-1, y_{i}\right)}{p\left(x_{\imath}, y_{\imath}\right)}=1 \text {, }
$$


and

$$
\frac{1}{n} \sum_{i=1}^{n} \frac{p\left(x_{\imath}, y_{i}-1\right)}{p\left(x_{\imath}, y_{\imath}\right)}=1
$$

$$
\frac{1}{n} \sum_{\imath=1}^{n} \frac{p\left(x_{i}-1, y_{i}-1\right)}{p\left(x_{\imath}, y_{\imath}\right)}=1 \text {, }
$$

where $p(k, l)=P(X=k, Y=l)$ with parameters $\lambda_{10}, \lambda_{01}$ and $\lambda_{11}$.

We usually call the relations the normal equalities.

Proof of the theorem. To maximize the likelihood function $\prod_{\imath=1}^{n} p\left(x_{\imath}, y_{\imath}\right)$ with respect to the parameters $\lambda_{10}, \lambda_{01}$ and $\lambda_{11}$, we have to differentiate the logarithm of likelihood function about the parameters and to put its value zero.

$$
\frac{\partial}{\partial \lambda_{10}} \prod_{\imath=1}^{n} p\left(x_{\imath}, y_{\imath}\right)=\sum_{\imath=1}^{n} \frac{\frac{\partial}{\partial \lambda_{10}} p\left(x_{\imath}, y_{\imath}\right)}{p\left(x_{\imath}, y_{\imath}\right)}-\prod_{\imath=1}^{n} p\left(x_{\imath}, y_{\imath}\right)=0 \text {, }
$$

where the differential of the probability density is given by a convenient relation

Because, we have

$$
\frac{\partial}{\partial \lambda_{10}} p(x, y)=p(x-1, y)-p(x, y) .
$$

$$
\begin{aligned}
\frac{\partial}{\partial \lambda_{10}} p(k, l) & =\frac{\partial}{\partial \lambda_{10}} \sum_{\substack{\beta+\delta=k \\
\gamma+\delta=l}} \frac{\lambda_{10}^{\beta} \lambda_{01}^{\gamma} \lambda_{11}^{\delta}}{\beta ! \gamma ! \delta !} e^{-\lambda_{10}-\lambda_{01}-\lambda_{11}} \\
& =\sum_{\substack{\beta+\delta=k \\
\gamma+\delta=l \\
\beta-1 \geq 0}} \frac{\lambda_{10}^{\beta-1} \lambda_{01}^{r} \lambda_{11}^{\delta}}{(\beta-1) ! \gamma ! \delta !} e^{-\lambda_{10}-\lambda_{01}-\lambda_{11}}-\sum_{\substack{\beta+\delta=k \\
\gamma+\delta=l}} \frac{\lambda_{10}^{\beta} \lambda_{01}^{\gamma} \lambda_{11}^{\delta}}{\beta ! \gamma ! \delta !} e^{-\lambda_{10}-\lambda_{01}-\lambda_{11}} \\
& =\sum_{\substack{\beta+\delta=k-1 \\
\gamma+\delta=l}} \frac{\lambda_{10}^{\beta} \lambda_{01}^{\gamma} \lambda_{11}^{\delta}}{\beta ! \gamma ! \delta !} e^{-\lambda_{10}-\lambda_{01}-\lambda_{11}-p(k, l)} \\
& =p(k-1, l)-p(k, l) .
\end{aligned}
$$

Therefore, we can verify,

$$
\begin{aligned}
\frac{\partial}{\partial \lambda_{10}} \prod_{\imath=1}^{n} p\left(x_{\imath}, y_{\imath}\right) & =\sum_{\imath=1}^{n} \frac{\frac{\partial}{\partial \lambda_{10}} p\left(x_{\imath}, y_{\imath}\right)}{p\left(x_{\imath}, y_{\imath}\right)} \prod_{\imath=1}^{n} p\left(x_{\imath}, y_{\imath}\right) \\
& =\sum_{\imath=1}^{n} \frac{p\left(x_{i}-1, y_{\imath}\right)-p\left(x_{\imath}, y_{\imath}\right)}{p\left(x_{\imath}, y_{\imath}\right)} \prod_{\imath=1}^{n} p\left(x_{\imath}, y_{\imath}\right) \\
& =\left[\sum_{\imath=1}^{n} \frac{p\left(x_{i}-1, y_{\imath}\right)}{p\left(x_{\imath}, y_{\imath}\right)}-n\right] \prod_{\imath=1}^{n} p\left(x_{\imath}, y_{\imath}\right) .
\end{aligned}
$$

The equivalent condition of the normal equation 
is given by

$$
\frac{\partial}{\partial \lambda_{10}} \prod_{\imath=1}^{n} p\left(x_{\imath}, y_{\imath}\right)=0
$$

$$
\sum_{\imath=1}^{n} \frac{p\left(x_{i}-1, y_{\imath}\right)}{p\left(x_{\imath}, y_{\imath}\right)}=n
$$

By using similar calculations about

and

$$
\frac{\partial}{\partial \lambda_{01}} \prod_{\imath=1}^{n} p\left(x_{\imath}, y_{\imath}\right)=0
$$

$$
\frac{\partial}{\partial \lambda_{11}} \prod_{\imath=1}^{n} p\left(x_{\imath}, y_{\imath}\right)=0,
$$

we will be given the equivalent conditions

and

$$
\sum_{\imath=1}^{n} \frac{p\left(x_{\imath}, y_{i}-1\right)}{p\left(x_{\imath}, y_{\imath}\right)}=n
$$

respectively.

$$
\sum_{\imath=1}^{n} \frac{p\left(x_{i}-1, y_{i}-1\right)}{p\left(x_{\imath}, y_{\imath}\right)}=n
$$

THEOREM 2. MLE of $\lambda_{10}, \lambda_{01}$ and $\lambda_{11}$ denoted as $\hat{\lambda}_{10}, \hat{\lambda}_{01}$ and $\hat{\lambda}_{11}$ satısfy $\hat{\lambda}_{10}+\hat{\lambda}_{11}$ $=\bar{x}$ and $\hat{\lambda}_{01}+\hat{\lambda}_{11}=\bar{y}$.

Proof of the theorem. From the first normal equation

and a relation:

$$
\sum_{i=1}^{n} \frac{p\left(x_{i}-1, y_{\imath}\right)}{p\left(x_{\imath}, y_{\imath}\right)}=n
$$

we have

$$
k p(k, l)=\lambda_{10} p(k-1, l)+\lambda_{11} p(k-1, l-1),
$$

and

$$
\sum_{\imath=1}^{n} \frac{p\left(x_{i}-1, y_{\imath}\right)-p\left(x_{\imath}, y_{\imath}\right)}{p\left(x_{\imath}, y_{\imath}\right)}=0
$$

$$
\lambda_{10} p\left(x_{\imath}-1, y_{\imath}\right)=x_{\imath} p\left(x_{\imath}, y_{\imath}\right)-\lambda_{11} p\left(x_{i}-1, y_{i}-1\right) .
$$

Then, the first normal equation is expressed as following:

$$
\begin{aligned}
& \sum_{i=1}^{n} \frac{\lambda_{10} p\left(x_{i}-1, y_{\imath}\right)-\lambda_{10} p\left(x_{\imath}, y_{\imath}\right)}{p\left(x_{\imath}, y_{\imath}\right)} \\
= & \sum_{i=1}^{n} \frac{x_{\imath} p\left(x_{\imath}, y_{\imath}\right)-\lambda_{11} p\left(x_{i}-1, y_{i}-1\right)-\lambda_{10} p\left(x_{\imath}, y_{\imath}\right)}{p\left(x_{\imath}, y_{\imath}\right)} \\
= & \sum_{i=1}^{n}\left[\left(x_{i}-\lambda_{10}\right)-\lambda_{11} \frac{p\left(x_{i}-1, y_{i}-1\right)}{p\left(x_{\imath}, y_{\imath}\right)}\right] \\
= & \sum_{i=1}^{n} x_{i}-n \lambda_{1},-\lambda_{11} \sum_{\imath=1}^{n} \frac{p\left(x_{i}-1, y_{i}-1\right)}{p\left(x_{\imath}, y_{\imath}\right)}=0,
\end{aligned}
$$


and by the third normal equation, we have

$$
\sum_{i=1}^{n} x_{i}-n\left(\lambda_{10}+\lambda_{11}\right)=0
$$

and a concluding equation

$$
\lambda_{10}+\lambda_{11}=\sum_{i=1}^{n} x_{2} / n=\bar{x} .
$$

Similarly, we have another concluding equation from the second and the third normal equations

$$
\lambda_{01}+\lambda_{11}=\sum_{\imath=1}^{n} y_{\imath} / n=\bar{y} .
$$

These two simple relations come only from the normal equations and $\lambda_{10}, \lambda_{01}$ and $\lambda_{11}$ must be symbolized by MLE $\hat{\lambda}_{10}, \hat{\lambda}_{01}$ and $\hat{\lambda}_{11}$.

Further calculation about $\hat{\lambda}_{10}, \hat{\lambda}_{01}$ and $\hat{\lambda}_{11}$ is very difficult and we have to calculate the individual estimator by numerical method. In the next section we shall treat the practical calculating method showing how to get MLE $\hat{\lambda}_{10}, \hat{\lambda}_{01}$ and $\hat{\lambda}_{11}$.

2-2. Practical calculation of MLE $\hat{\lambda}=\left(\hat{\lambda}_{10}, \hat{\lambda}_{01}, \hat{\lambda}_{11}\right)$.

THEOREM 3. MLE $\hat{\lambda}_{10}, \hat{\lambda}_{01}$ and $\hat{\lambda}_{11}$ satısfy the relations

$$
\hat{\lambda}_{10}+\hat{\lambda}_{11}=\bar{x}, \quad \hat{\lambda}_{01}+\hat{\lambda}_{11}=\bar{y} \quad \text { and } \quad 0 \leqq \hat{\lambda}_{11} \leqq \min (\bar{x}, \bar{y})
$$

which maximize the logarithm of likelihood function $\log \prod_{\imath=1}^{n} p\left(x_{\imath}, y_{\imath}\right)$.

Proof of the theorem. This theorem is a representation of the last theorem for our calculation of MLE. Denote $m=\min (\bar{x}, \bar{y})$ for simplicity of notation, $\lambda_{10}$, $\lambda_{01}$ and $\lambda_{11}$ are nonnegative parameters, so that we have $0 \leqq \hat{\lambda}_{11} \leqq m$. To get $\hat{\lambda}_{11}$ we have to move $\lambda_{11}$ in the interval $[0, m]$ which maximize the logarithm of likelihood function $l l f$.

To calculate MLE $\hat{\lambda}_{10}, \hat{\lambda}_{01}$ and $\hat{\lambda}_{11}$ numerically, we have to compare the value of llf on the scanning space of the three parameters. This theorem states that we may find MLE which maximizes the value of $l l f$ in one dimensional parameter space, that is, our scanning space of the parameters reduce to one dimensional subspace ;

$$
\lambda_{10}=\bar{x}-\lambda_{11}, \quad \lambda_{01}=\bar{y}-\lambda_{11} \quad \text { and } \quad \lambda_{11} \in[0, m] .
$$

At the begining of computation, we compare the value llf in the rule $\lambda_{11}=0.0,1.0,2.0, \cdots \leqq m=(\bar{x}, \bar{y})$ and $\lambda_{10}=\bar{x}-\lambda_{11}, \lambda_{01}=\bar{y}-\lambda_{11}$, that is, $\lambda_{11}$ moves on nonnegative integers from 0 to the integer lower than $m$. We will find one $\lambda_{11}$ which maximizes llf or two $\lambda_{11}$ which maximize the function. Usually we get only one $\lambda_{11}$ which maximizes llf and occasionally we look for two $\lambda_{11}$ which 
maximize the function. In the first case we have to look for $\lambda_{11}$ in the interval of both sides of $\lambda_{11}$ which maximizes the function and we have adjusted the scanning step in the reduced parameter space to one quater of preceding scanning step 1.0.
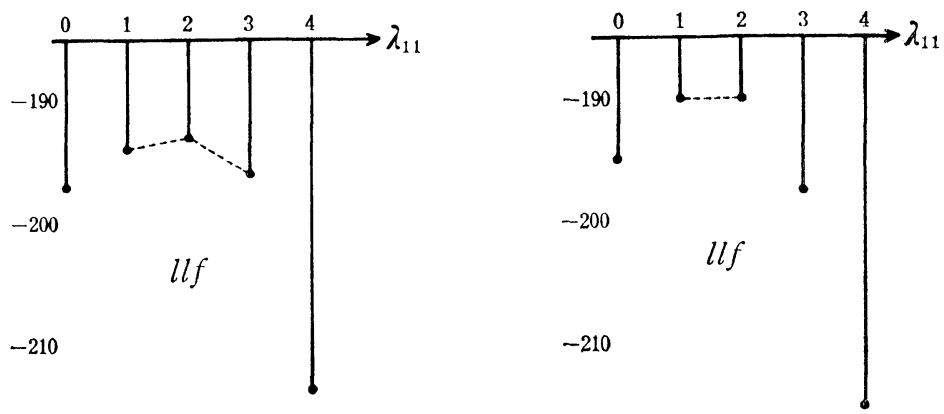

Fig. 1

2-3. Automatic reduction rule of $\lambda_{11}$ scanning space.

Let us discuss in detail the reduction rule. We put the scanning space of $\lambda_{11}$ as $D_{0}$ initially,

$$
D_{0}=\left\{0,1,2, \cdots, m_{0}\right\},
$$

where $m_{0}$ equals to the maximum integer lower than $m=\min (\bar{x}, \bar{y})$.

(case 1) $\lambda_{11}=0$ maximizes llf in the scanning space $D_{0}$.

(case 2) One of $\lambda_{11}=k$ in $1,2, \cdots, m_{0}-1$ maximizes llf in the scanning space $D_{0}$.

(case 3) Two of $\lambda_{11}=k, k+1$ in $0,1,2, \cdots, m_{0}$ maximize llf in the scanning space $D_{0}$.

(case 4) $\lambda_{11}=m_{0}$ maximizes $l l f$ in the scanning space $D_{0}$.

Scanning $\lambda_{11} \in D_{0}$ as to maximize $l l f$, we have four cases (case 1 ), $\cdots$, (case 4). In every case, to compute MLE $\hat{\lambda}_{11}$ more detail, we have to reduce the scanning space and the scanning step. We have used the secondary scanning step as one quater of the preceding one. Then we have a new scanning space $D_{\mathrm{l}}$ as following :

(case 1) $D_{1}=\left\{0, \frac{1}{4}, \frac{2}{4}, \frac{3}{4}, 1\right\}$

(case 2) $D_{1}=\left\{k-1, k-1+\frac{1}{4}, k-1+\frac{2}{4}, k-1+\frac{3}{4}, k, k+\frac{1}{4}, k+\frac{2}{4}, k+\frac{3}{4}, k+1\right\}$

(case 3) $D_{1}=\left\{k, k+\frac{1}{4}, k+\frac{2}{4}, k+\frac{3}{4}, k+1\right\}$

(case 4) $D_{1}=\left\{m_{0}-1, m_{0}-1+\frac{1}{4}, m_{0}-1+\frac{2}{4}, m_{0}-1+\frac{3}{4}, m_{0}, m_{0}+\frac{1}{4}, \cdots, m_{0}+\frac{t}{4}\right\}$ 
where $t$ is the maximum integer in $0,1,2,3$ such that

$$
m_{0}+\frac{t}{4} \leqq m=\min (\bar{x}, \bar{y}) .
$$

In the primary step, we compare the value of llf by $\lambda_{11}$ scanning only the integral values on $[0, m]$, as to find $\lambda_{11}$ which maximizes llf. Secondary step, if we find one $\lambda_{11}$ maximizing $l l f$ as (case 2), we can reduce the scanning interval $[0, m]$ to $[k-1, k+1]$ where $k$ is the value of $\lambda_{11}$ maximizing llf. If we find one $\lambda_{11}=0$ maximizing $l l f$ as (case 1 ), we can reduce the scanning interval to $[0,1]$ and if we find one $\lambda_{11}=m_{0}$ maximizing llf as (case 4), we can reduce the scanning interval to $\left[m_{0}-1, m\right]$. If we find two $\lambda_{11}=k, k+1$ $\left(k=0,1, \cdots, m_{0}-1\right)$ maximizing llf we can reduce the scanning interval to $[k, k+1]$ as (case 3). Our scanning of $\lambda_{11}$ is made in the reduced interval and the scanning step is adjusted to a quater step of preceding scanning step, where the scanning step of $\hat{\lambda}_{11}$ in the reduced interval may change under another reduction rule and the total computing time will change. Then we can reduce our scanning space $D_{0}$ to $D_{1}$ as denoted above.

Under this inductive routine, if we set 0.001 as the exactness of the calculation of MLE $\hat{\lambda}_{11}$, then we will obtain MLE $\hat{\lambda}_{11}$ involving the exactness after high resolution computing method.

\section{Section 3. A sequence of random numbers from $P(\lambda)$ and computation of MLE $\hat{\lambda}_{11}$ by computer.}

In this section a result of computer simulation will be demonstrated, one is a change of llf under our reduced linear space, and the other is a computing process of finding MLE $\hat{\lambda}_{11}$.

3-1. Simulation of a sequence of bivariate Poisson distribution $P(\lambda)$.

Given parameters $\lambda_{10}, \lambda_{01}$ and $\lambda_{11}, X_{10}, X_{01}$ and $X_{11}$ are independent univariate Poisson distributions then $(X, Y)$ from a bivariate Poisson distribution is expressed by

$$
X=X_{10}+X_{11} \text { and } Y=X_{01}+X_{11} \text {. }
$$

To make a sequence of random numbers of the distribution $P(\lambda)$, we should make three series of independent univariate Poisson random variables $X_{10}, X_{01}$ and $X_{11}$.

Following figure is our flow-chart of making bivariate Poisson random variables. 


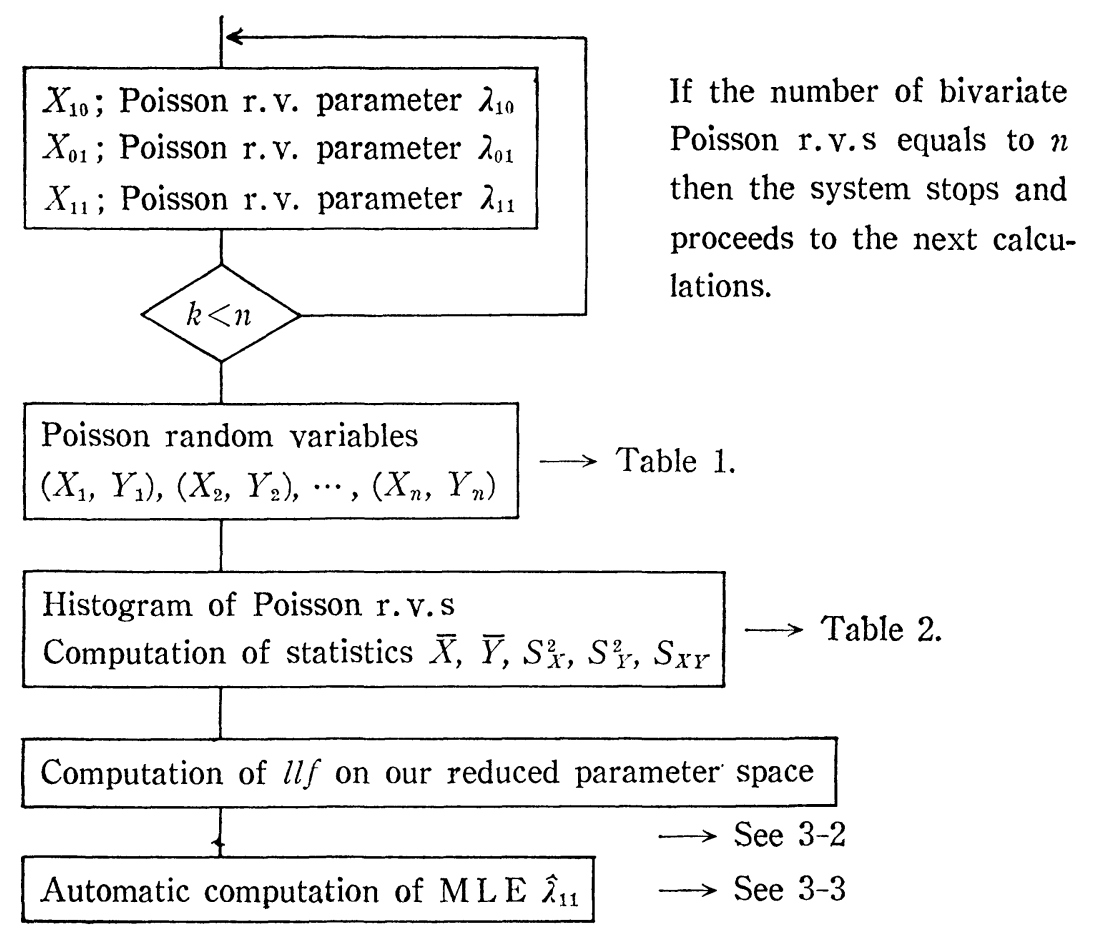

Fig. 2

Poisson random variables with parameters $\lambda_{10}=3.0, \lambda_{01}=3.0$ and $\lambda_{11}=2.0$

$\begin{array}{llllllllll}(6,5) & (9,5) & (2,4) & (7,3) & (6,4) & (6,4) & (9,7) & (9,7) & (1,3) & (4,6) \\ (5,7) & (3,7) & (3,4) & (3,3) & (4,5) & (4,4) & (5,7) & (5,5) & (1,6) & (6,10) \\ (4,3) & (6,2) & (5,6) & (5,6) & (6,2) & (6,6) & (3,5) & (3,2) & (5,7) & (1,4) \\ (3,3) & (7,5) & (6,5) & (6,4) & (2,3) & (7,5) & (6,3) & (3,3) & (6,2) & (4,6) \\ (1,5) & (9,10) & (5,6) & (4,5) & (10,2) & (4,2) & (7,4) & (5,8) & (4,4) & (1,1) \\ (5,2) & (7,9) & (4,7) & (4,4) & (4,8) & (7,4) & (5,5) & (5,5) & (4,6) & (11,13) \\ (5,3) & (6,6) & (3,6) & (8,11) & (7,9) & (5,8) & (4,3) & (5,5) & (2,7) & (6,7) \\ (3,1) & (6,8) & (4,5) & (12,10) & (2,2) & (8,3) & (8,9) & (6,2) & (9,7) & (3,4) \\ (5,2) & (4,9) & (5,8) & (4,7) & (9,7) & (5,3) & (4,1) & (7,7) & (3,1) & (4,4) \\ (1,5) & (1,6) & (3,1) & (4,8) & (7,6) & (3,4) & (7,4) & (2,2) & (1,1) & (4,3)\end{array}$


Histogram of the Poisson random variables

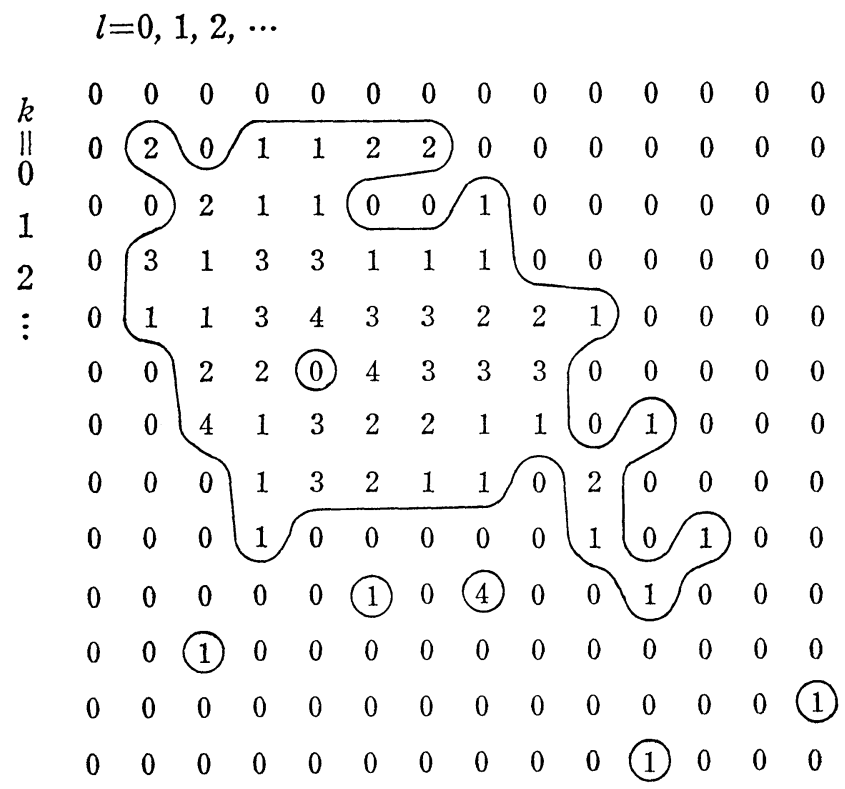

Statistics based on the Poisson random variables.

$$
\begin{gathered}
\qquad \begin{array}{c}
\bar{X}=4.93, \quad S_{X}^{2}=5.3251 \\
\bar{Y}=5.03, \quad S_{Y}^{2}=6.1891 \\
S_{X Y}=2.5021
\end{array} \\
\text { where } S_{X Y}=\sum_{\imath=1}^{n}\left(X_{i}-\bar{X}\right)\left(Y_{\imath}-\bar{Y}\right) / n .
\end{gathered}
$$

Table 2

3-2. The change of llf under our scanning rule.

Our reduced one dimensional parameter space is expressed as

$$
\lambda_{10}=\bar{X}-\lambda_{11}, \quad \lambda_{01}=\bar{Y}-\lambda_{11} \quad \text { and } \lambda_{11} \in[0, m]
$$

where $m=\min (\bar{x}, \bar{y})$. From $\bar{x}=4.93, \bar{y}=5.03$, we get $m=4.93$ so that our reduced parameter space is expressed;

$$
\lambda_{10}=4.93-\lambda_{11}, \quad \lambda_{01}=4.93-\lambda_{11} \text { and } \lambda_{11} \in[0,4.93] .
$$

We tried to calculate llf on the space under a scanning step 0.1 of the third parameter $\lambda_{11}$. Next table and graph explain a relation of variable $\lambda_{11}$ and $l l f$. And we will find MLE of $\lambda_{11}$ close to $\lambda_{11}=1.9$. 


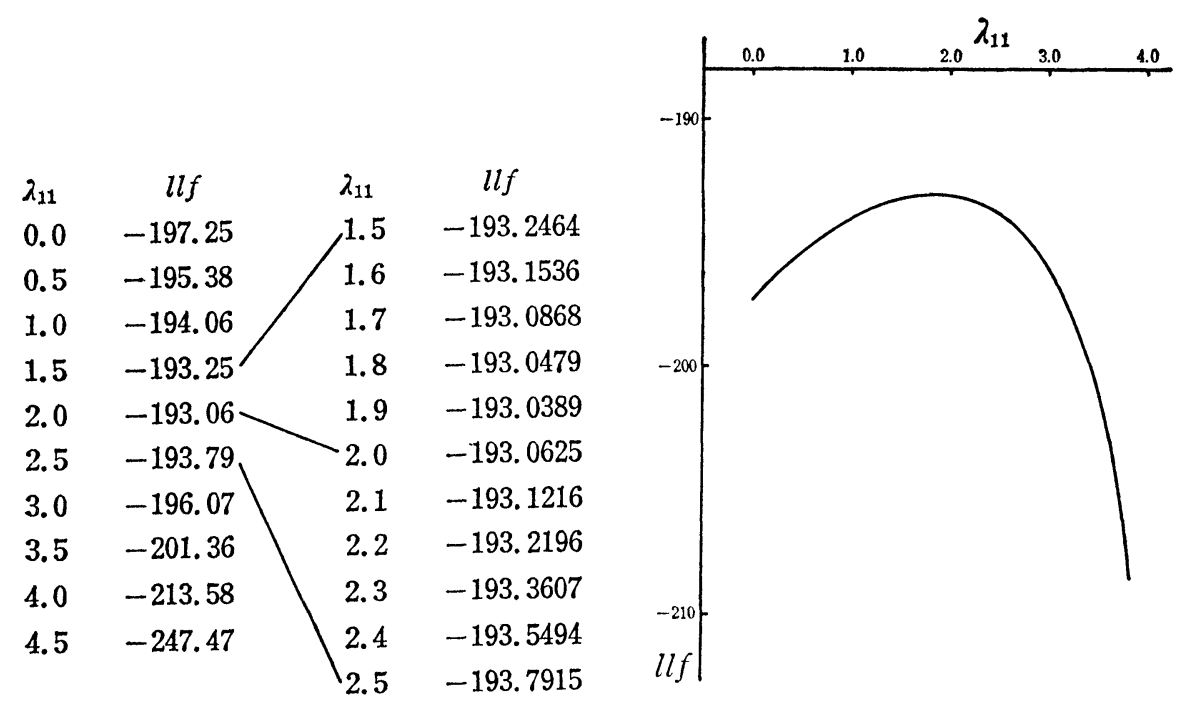

Table 3

Fig. 3

3-3. Automatic computing process of MLE $\hat{\lambda}_{11}$.

If we would like to know the function of our automatic computing process of MLE $\hat{\lambda}_{11}$, we can easily pull out the scanning spaces $D_{0}, D_{1}, \cdots$ and llf values on each spaces. Following table and graph explain the function.

Lower limit

Upper limit

Scan

$\begin{array}{lrl}D_{0} & 0-4 & 1 \\ D_{1} & 1-3 & 1 / 4 \\ D_{2} & 1+3 / 4-1+5 / 4 & 1 / 16 \\ D_{3} & 1+3 / 4+1 / 16-1+3 / 4+3 / 16 & 1 / 64 \\ D_{4} & 1+3 / 4+1 / 16+3 / 64-1+3 / 4+1 / 16+5 / 64 & 1 / 256 \\ D_{5} & 1+3 / 4+1 / 16+3 / 64+4 / 256-1+3 / 4+1 / 16+3 / 64+6 / 256 & 1 / 1024 \\ D_{6} & 1+3 / 4+1 / 16+3 / 64+4 / 256-1+3 / 4+1 / 16+3 / 64+4 / 256 & \\ & +3 / 1024 & +5 / 1024\end{array}$

Table 4 
Graph of upper and lower limits of reduced scanning spaces $D_{0}, D_{1}, \cdots$.

Fig. 4

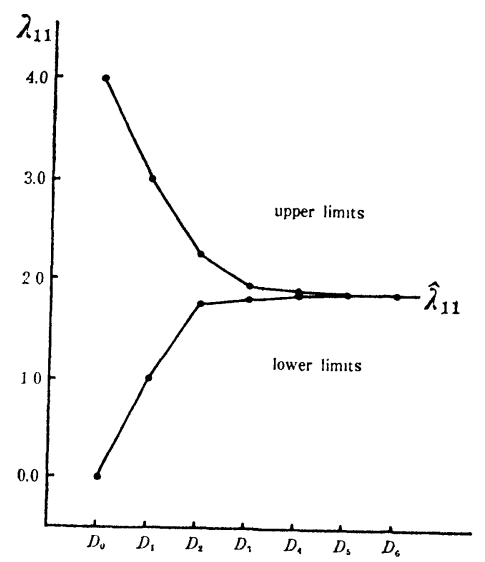

After our automatic computing process of MLE, we get MLE $\hat{\lambda}_{11}=1.879$ and from the assertion of theorem 3 , we get MLE $\hat{\lambda}_{10}=4.93-1.879=3.051$ and MLE $\hat{\lambda}_{01}=5.03-1.879=3.151$. These are the maximum likelihood estimators of the bivariate Poisson random variables simulated by computer. We have another estimator for $\lambda_{11}$,

$$
S_{X Y}=\sum_{\imath=1}^{n}\left(X_{i}-\bar{X}\right)\left(Y_{i}-\bar{Y}\right) / n=\sum_{\imath=1}^{n} X_{\imath} Y_{\imath} / n-\bar{X} \bar{Y}=2.5021 .
$$

The parameter used to the simulation was $\lambda_{10}=3.0, \lambda_{01}=3.0$ and $\lambda_{11}=2.0$. And our MLE is expressed as $\hat{\lambda}_{10}=3.051, \hat{\lambda}_{01}=3.151$ and $\hat{\lambda}_{11}=1.879$. Another estimation for $\lambda_{11}$ is given by $S_{X Y}=2.5021$.

Remark. The aim of the last section is to answer the questions: how to make the bivariate Poisson random variables (by simulation) and how to calculate MLE of the parameter $\lambda=\left(\lambda_{10}, \lambda_{01}, \lambda_{11}\right)$. But the next question to answer would be how to check the fitness of the bivariate Poisson distribution for given bivariate data $\left(x_{1}, y_{1}\right),\left(x_{2}, y_{2}\right), \cdots,\left(x_{n}, y_{n}\right)$ as given in table 1 .

\section{REFERENCES}

[1] Kawamura, K., The structure of bivariate Poisson distribution, Kōdai Math. Sem. Rep., 25, No. 2, (1973).

[2] Polak, M., Poisson approximation for sums of independent bivariate Bernoulli vectors, Kodal Math. J., 5, No. 3, (1982).

[3] Johnson, N. L. And Kotz S., Discrete distributions, Houghton Mifflin Co. (1969).

[4] Holgate, P., Estimation for the bivaliate Poisson distribution, Biometrika, 51, (1964), 241-245. 